Н. В Федорова

\title{
ИНТЕЛЛЕКТУАЛЬНЫЙ ИНСТРУМЕНТ УПРАВЛЕНИЯ ТРАФИКОМ В МУЛЬТИСЕРВИСНОЙ МАКРОСЕТИ
}

Аннотация. Приведены основные подходы контроля использования трафика в мультисервисной макросети. Рассмотрен принцип работы глубокого анализа пакетов. Предложена новая модель сервисов и использование стратегии “персональный тариф персональный пакет сервисов”. А также предложена система управления: сервис маршрут - тариф.

Ключевые слова: мультисервисная макросеть, контроль трафика, глубокий анализ пакетов, персональный пакет сервисов, персональный тариф.

\section{Введение}

Активное развитие и функционирование технологий, соединяющих общество в едином пространстве, способствует и ведет к созданию мультисервисной макросети оператора. При интеграции в существующую сеть оператора, на первый план выходит взаимодействие: оператор сервисов - пользователь сервисов. Для эффективного функционирования в цифровом пространстве необходимо максимально глубокое понимание поведенческих особенностей и предпочтений отдельных пользователей, которое следует демонстрировать на каждом этапе взаимодействия $[1,2]$.

\section{Постановка задачи}

В последнее время объемы трафика стремительно увеличиваются с каждым годом в огромных масштабах, заставляя оператора сервисов постоянно наращивать пропускную мощность. При обеспечении качественного сервиса, и постоянно растущих потребности клиента оператор должен контролировать и управлять растущими аппетитами пользователей $[2,3]$.

Для контроля использования трафика пользователями сейчас практикуется несколько основных подходов:

1. Ограничение ежемесячного объема трафика. Такое решение малопривлекательно для пользователя и исходя из этого не популярно. Используется такой метод в основном для предоставления услуг наиболее дорогого мобильного Интернета. Оператор может ограничить трафик в добровольно-принудительном порядке - не лишая выбравшего свой лимит пользователя услуги совсем, а просто понижая скорость до критического минимума. Но это не решает проблемы с пропускной способностью до того времени, пока пользователи не использовали свой лимит.

2. Ограничение полосы пропускания. Этот вариант сейчас наиболее распространен, но, к сожалению, малоэффективен: слишком сильное ограничение вызовет отток клиентов, а малое - не решит проблемы.

(C) Н. В Федорова 
Такие подходы на сегодня не удовлетворяют ни пользователей, ни операторов. Единственным логичным выходом их сложившейся ситуации является контроль на уровне приложений, в случае которого лимитируется не сам трафик или его объемы, а контролируется его использование. И в этом помогает DPI (Deep Packet Inspection) анализ.

\section{Основная часть}

Система DPI [4] выполняет глубокий анализ всех проходящих через неё пакетов. Термин «глубокий» подразумевает анализ пакета на верхних уровнях модели OSI (Open Systems Interconnection), а не только по стандартным номерам портов. Помимо изучения пакетов по неким стандартным паттернам, по которым можно однозначно определить принадлежность пакета определённому приложению, скажем, по формату заголовков, номерам портов и т.п., система DPI осуществляет и так называемый поведенческий анализ трафика, который позволяет распознать приложения, не использующие для обмена данными заранее известные заголовки и структуры данных.

Важной отличительной особенностью настоящего DPI является возможность аналитики трафика за счёт сбора различного рода статистики с разбивкой по приложениям, по тарифным планам, по регионам, по типам абонентских устройств и т.д.

Система DPI [4], как правило, устанавливается на границе сети оператора в разрыв существующих аплинков, уходящих от пограничных маршрутизаторов. Тем самым, весь трафик, который покидает или входит в сеть оператора, проходит через DPI, что даёт возможность его мониторинга и контроля. Для решения специфических задач можно устанавливать эту систему не на границе сети, а спускать её ниже, ближе к конечным пользователям. Это может быть полезно тем операторам, которые по ряду причин помимо утилизации внешних каналов также хотят решать задачу контроля внутренних. Естественно, здесь речь идёт о достаточно крупных операторах сервисов с большой распределённой сетью масштабов страны и с достаточно дорогими канальными ёмкостями.

\section{Новая модель сервисов}

Глядя на то, как подписчики пользуются купленной ими полосой, какие приложения используют, оператор может изучать потребности каждой категории подписчиков и предлагать им более гибкие и совершенные тарифные планы.

Подход очень интересный, и выгодный как для пользователя, так и для оператора. Тенденции развития телекоммуникационного рынка таковы, что для операторов продавать трубу, как они делают сейчас, скоро будет просто невыгодно. Задача DPI [5] в данном разрезе - реализовать новые модели предоставления услуг конечному пользователю. Некоторые мировые операторы маленькими шагами уже двигаются к данной идее. 
Важным критерием при создании новой модели сервисов является модель управления сетевыми ресурсами и формированием тарифного плана пользователей, учитывая требования к сервису на основе производительности (рис. 2).

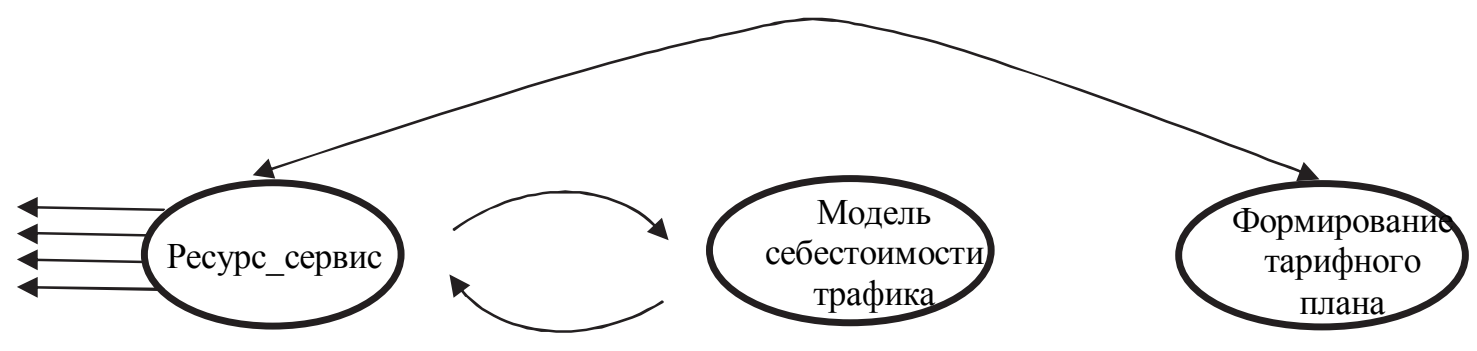

Рис. 2. Модель управления сетевыми ресурсами и формированием тарифного плана пользователей

В настоящее время тариф на услуги связи, который используется, является “сплошным” и ни о каком “принципе справедливости” речь не идет, т.к. скорость работы зависит только от загрузки канала, а не от объема скаченного трафика. При появлении мультисервисной макросети необходим переход на персональный тариф с выгодами для оператора и пользователя.

“Мир меняется! Все становится сервисом". Используя современные технологии 2G, 3G, 4G, 5G [6, 7] как сервис TaaS (Technology as a Service) пользователю хочется видеть индивидуальный подход. Для формирования индивидуального предложения каждому пользователю мультисервисной макросети необходимо создание единой системы для управления задачами: “персональный тариф - персональный пакет сервисов”. На рис. 2 показан пример персонального пакета сервисов.

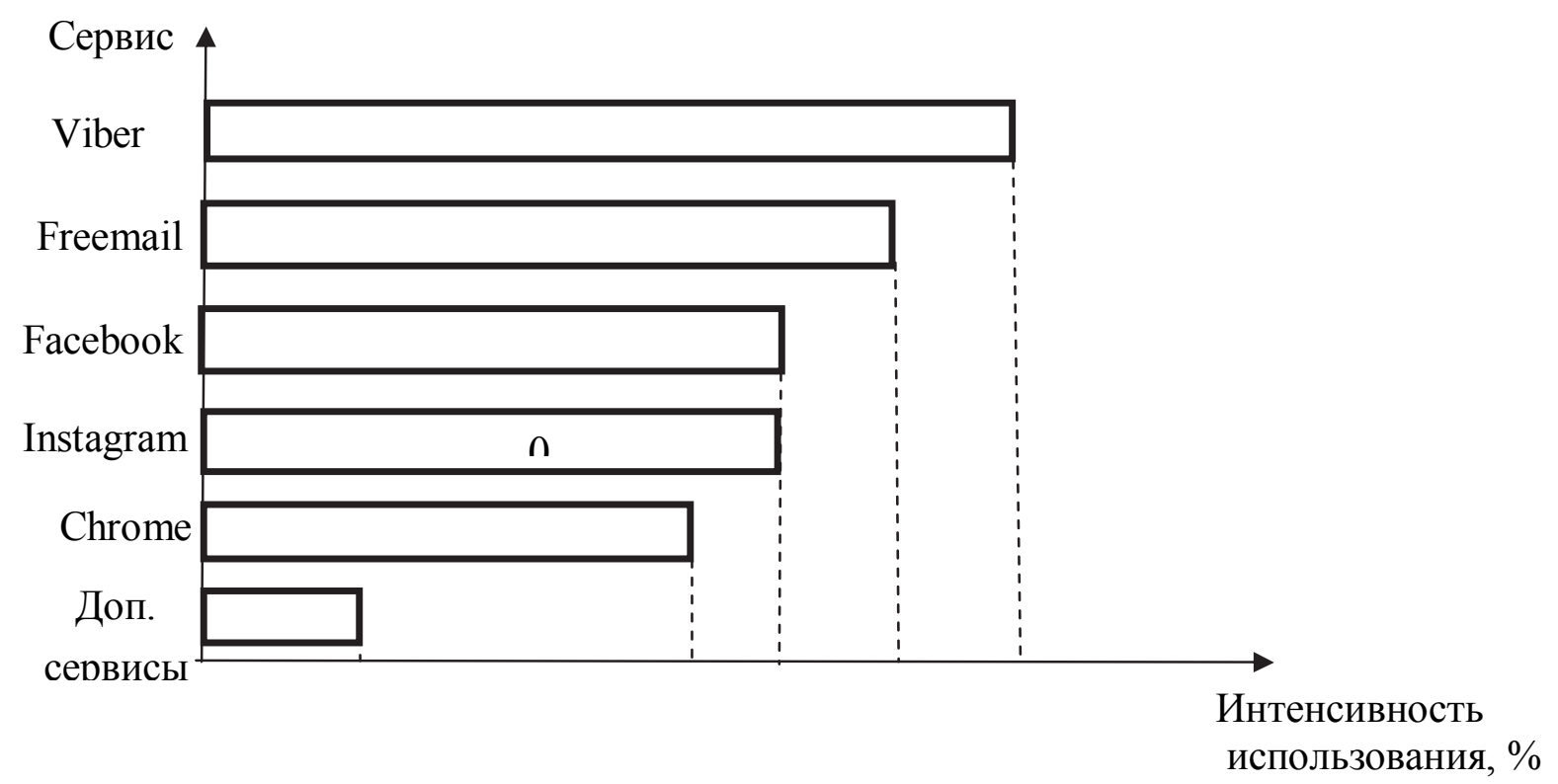

Рис. 3. Персональный пакет сервисов 
На рис. 3 показано управление и взаимосвязь между сервисом пользователя, маршрутом сервиса пользователя и тарифом сервиса пользователя.

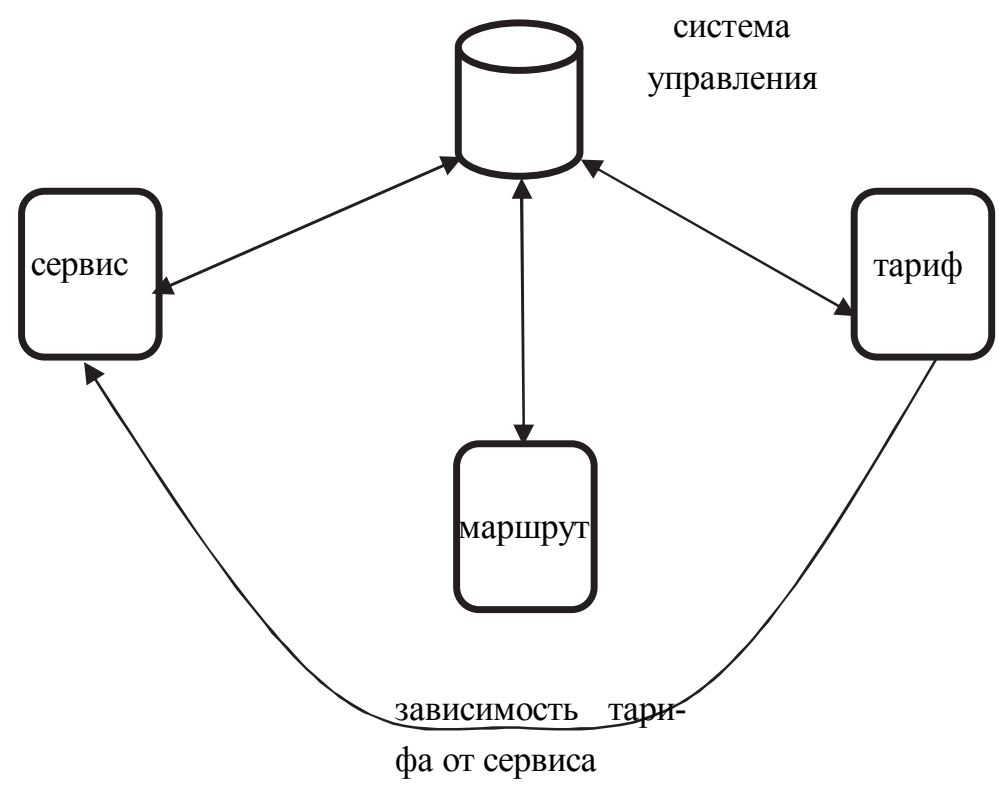

Рис. 3 Система управления: сервис - маршрут - тариф

Система управления, показанная на рис. 3 объединяет широкий спектр сервисов технологий $2 \mathrm{G}, 3 \mathrm{G}, 4 \mathrm{G}, 5 \mathrm{G}$, маршруты доставки сервисов и формирует тарифы на сервисы согласно профилю пользователя, в одном решении, работающем по так называемой модели TaaS.

Модель TaaS является производной от общепринятой модели $\mathrm{SaaS}$ (Software as a Service - программное обеспечение как сервис). Ключевым моментом концепции SaaS является отказ от традиционной модели установки программного обеспечения у заказчика. Отныне за его инсталляцию, обеспечение высокой готовности, своевременное обновление версий и т.п. отвечает оператор сервисов $[8,9]$.

Различия между моделями $\mathrm{SaaS}$ и TaaS состоят в том, что модель TaaS для формирования персонального тарифа также разграничивает данные разных пользователей и обеспечивает их надежную защиту, но необходимость совместного доступа пользователей к коду в модели TaaS отсутствует.

Модель TaaS [9] предполагает гибкие схемы оплаты предоставляемых сервисов. На практике пока встречаются случаи, когда клиент вносит фиксированный платеж за услугу вперед на продолжительный период, но все большее распространение получают схемы, при которых оплата производится помесячно, да еще с учетом числа пользователей, которые работали с тем или иным приложением, продолжительностью их работы и востребованной сетевой производительностью. В результате у оператора сервисов появляется возможность контролировать и регулировать уровень текущих расходов пользователя. Более того, по 
мере развития бизнеса изменения бизнес-процессов или ситуации на рынке оператор сервисов имеет возможность предложить своим пользователям новые функции, доступные в рамках используемого TaaS-решения, либо, напротив, аннулировать возможности, потребность в которых отпала.

\section{Выводы}

1. Активное развитие и функционирование технологий, соединяющих общество в едином пространстве, способствует и ведет к созданию мультисервисной макросети оператора.

2. При обеспечении качественного сервиса, и постоянно растущих потребности клиента оператор должен контролировать и управлять растущими аппетитами пользователей.

3. Важным критерием при создании новой модели сервисов является модель управления сетевыми ресурсами и формированием тарифного плана пользователей, учитывая требования к сервису на основе производительности

4. Первое место в стратегии “персональный тариф - персональный пакет сервисов” занимает потребность оператора телекоммуникаций взаимодействовать с конкретными пользователями. Объединяя сетевые ресурсы и возможности предоставления соответствующих сервисов, подкрепляя их индивидуальным подходом к пользователю, операторы телекоммуникаций станут придавать коммуникациям более клиенто-ориентированный характер.

5. Применение на практике операторами сервисов модели TaaS позволит перейти к форме персонального тарифа, а также разграничить данные разных пользователей и обеспечить их надежную защиту.

6. Модель TaaS предполагает гибкие схемы оплаты предоставляемых сервисов $[8,9]$ :

- клиент вносит фиксированный платеж за услугу вперед на продолжительный период;

- оплата производится помесячно с учетом числа пользователей, которые работали с тем или иным приложением, продолжительностью их работы и востребованной сетевой производительностью.

\section{Список использованных источников}

1. Прыжки через G: 3G - 4G - 5G / [Электронный ресурс]: Герман Богапов // Газета “Зеркало недели. Украина” № 15, 2016.

2. M.Mouly, M.B.Pautet. The GSM System for Mobile Communications. 1992. p.p. 702.

3. Тихвинский В. О., Терентьев С. В., Юрчук А. Б. Сети мобильной связи LTE: технология и архитектура. - М.: Эко-Трендз, 2010.

4. Глубокий анализ пакетов (DPI) как инструмент управления трафиком. Обзоры / [Электронный ресурс]: http://itc.ua/articles/glubokiy-analiz-paketov-dpi-kak-instrument-upravleniya-trafikom/. 
5. Краткий обзор технологии DPI — Deep Packet Inspection. Администрирование. - Сетевые технологии / [Электронный ресурс]: https://m.habrahabr.ru/post/111054/.

6. Технологии мобильной связи пятого поколения $(5 \mathrm{G})$. White Paper. - Ericsson, Сентябрь 2013: http"//ericsson.com/kz/news/130919_wp_5g_254740124.

7. Ю.А. Громаков. Сотовые системы подвижной радиосвязи. Технологии электронных коммуникаций. Том 48. "Эко-Трендз". Москва. 1994.

8. Компания Teradata запускает централизованный коммуникационный узел для полноценного персонализированного маркетинга / [Электронный pecypc]: http://www.retailloyalty.org/news/kompaniya-teradata-zapuskaet-tsentralizovannyy-kommunikatsionnyy-uzel-dlyapolnotsennogo-personalizi.

9. Что такое SaaS? / [Электронный pecypc]: http://clever-as.ru/articles/saas.html. 